\title{
Global Precipitation Measurement (GPM) mission core spacecraft systems engineering challenges
}

\author{
David J. Bundas ${ }^{* a}$, Deborah O’Neill ${ }^{\mathrm{a}}$, Michael Rhee ${ }^{\mathrm{a}}$, Thomas Feild ${ }^{\mathrm{a}}$, \\ Gary Meadows ${ }^{b}$, Peter Patterson ${ }^{b}$ \\ ${ }^{a}$ Mission Engineering and Systems Analysis Division, \\ Goddard Space Flight Center, Greenbelt, MD 20771 \\ ${ }^{\mathrm{b}}$ Swales Aerospace, 5050 Powder Mill Road, Beltsville, MD 20705
}

\begin{abstract}
The Global Precipitation Measurement (GPM) Mission is a collaboration between the National Aeronautics and Space Administration (NASA) and the Japanese Aerospace Exploration Agency (JAXA), and other US and international partners, with the goal of monitoring the diurnal and seasonal variations in precipitation over the surface of the earth. These measurements will be used to improve current climate models and weather forecasting, and enable improved storm and flood warnings. This paper gives an overview of the mission architecture and addresses the status of some key trade studies, including the geolocation budgeting, design considerations for spacecraft charging, and design issues related to the mitigation of orbital debris.
\end{abstract}

Keywords: spacecraft, systems engineering, precipitation

\section{INTRODUCTION}

The GPM Mission [1] plays a key role in the National Aeronautics and Space Administration's (NASA) Earth System Science (ESS) Research Strategy by providing measurements crucial to answering key questions related to the Earth's water and energy cycle (http://gwec.gsfc.nasa.gov). The GPM mission will provide this data by extending and improving the measurements made by the successful Tropical Rainfall Measurement Mission (TRMM) [2].

The key elements of the GPM mission are its constellation of spacecraft systems, including a Core Observatory, a Constellation Observatory, and partner-provided observatories; a ground validation system and a global data management information system for collecting, archiving, processing, and disseminating precipitation data to the user community. The NASA-provided Core Observatory carries the reference standard instrument set for a constellation of satellites carrying passive microwave radiometers. Instrumentation for the Core Observatory includes the Dualfrequency Precipitation Radar (DPR), provided by Japanese Aerospace Exploration Agency (JAXA), and a GPM Microwave Imager (GMI), being built by Ball Aerospace and Technologies Corporation. NASA's Goddard Space Flight Center (GSFC) provides GPM Mission project management and systems engineering. The project is currently in the formulation phase, having successfully completed a System Definition Review (SDR) in December 2005.

\section{Mission ARChitecture/Operations CONCEPT}

The GPM Mission Architecture (see Figure 1) consists of a constellation of satellites that provides continuous monitoring of precipitation over the globe. The GPM project provides two of these observatories, which are designated the Core Observatory and the Constellation Observatory. The remainder of the satellites in the constellation consist of other existing and planned US government resources (e.g., NPOESS), as well as contributed resources from international partners (e.g., Megha-Tropiques). Each of these missions contributes key passive microwave radiometer data, enabling the GPM mission to achieve global coverage. 
Instrumentation flying on the Core Observatory consists of the DPR and GMI instruments. The electronically-scanned DPR provides a 3-dimensional mapping of precipitation over both sea and land, and is used to calibrate GMI, as well as the passive microwave radiometers flying on other constellation spacecraft. The conically-scanned GMI provides precipitation rate information over a much wider swath than DPR, and its data contributes to estimates of global precipitation rates.

Science data from the GMI is continuously relayed via the TDRSS Demand Access System (DAS) to the Mission Operations Center (MOC) at GSFC, while science data from the DPR is stored and downlinked through scheduled TDRSS S-band Single Access (SSA) return link contacts once per orbit (approximately every 90 minutes). The GPM Precipitation Processing System (PPS) collects science data from the GPM constellation of satellites, processes it and provides products to the user community. A separate Ground Validation System (GVS) is being developed to provide information for the improved accuracy of modeling based on ground-based precipitation measurements made at various sites around the world.

\subsection{Ground System Architecture}

The GPM Ground System consists of three major items, all NASA provided: the Mission Operations Center (MOC), the Precipitation Processing System (PPS), and the Ground Validation System (GVS).

The MOC, developed and based at GSFC provides for control and monitoring of the two NASA-provided spacecraft. It

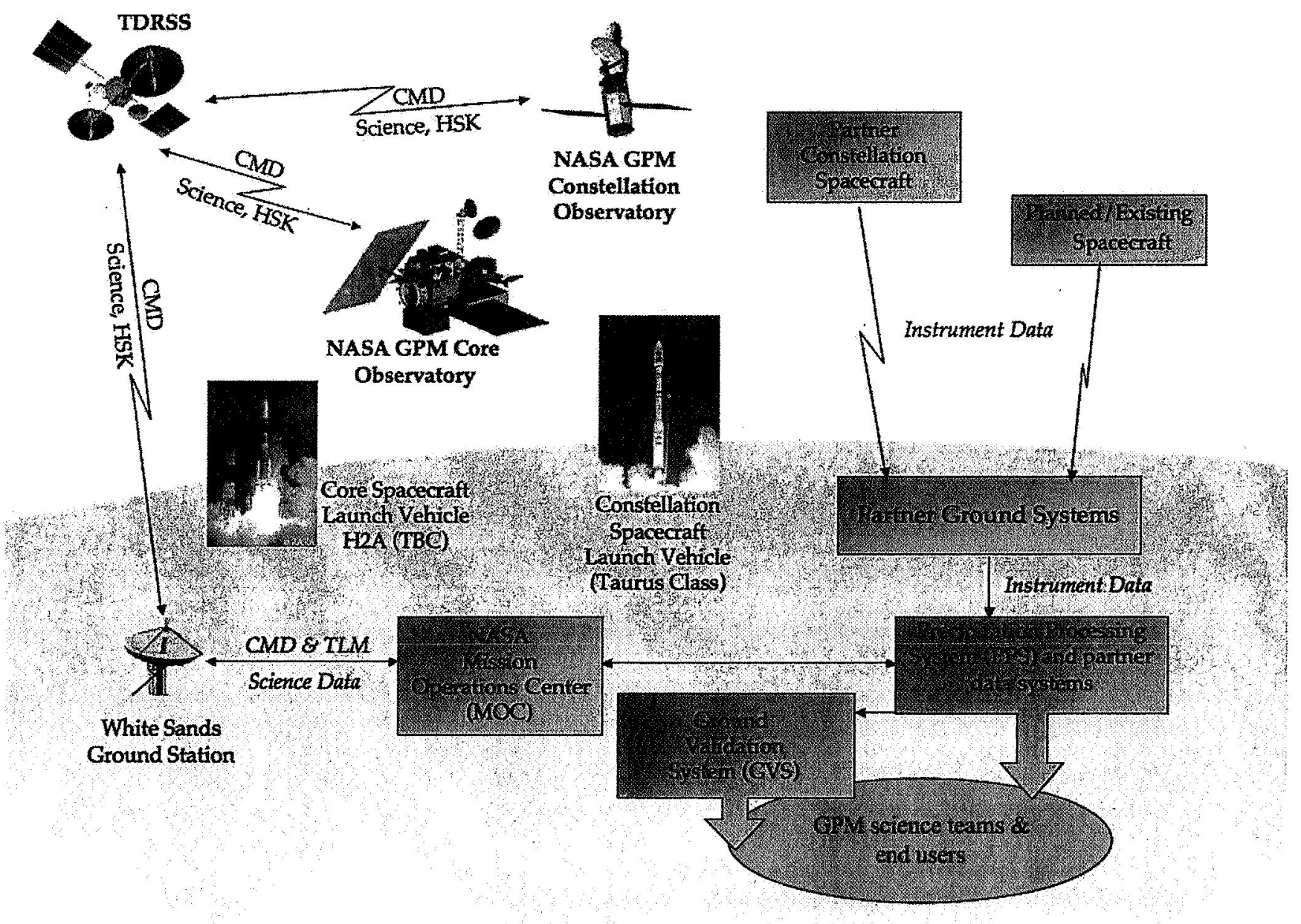

Figure 1. GPM Mission Architecture 
is responsible for maintaining the health and safety of the spacecraft, as well as managing the flow of science data from the satellites to the PPS. Once per orbit, a command load is relayed through White Sands Complex to the spacecraft via a TDRSS SSA link. Continuous monitoring of spacecraft health and safety is provided via the TDRSS Demand Access System (DAS). Science data from the GMI is provided via DAS every 5 minutes to satisfy data product latency requirements. The large data files produced by DPR are downloaded via an SSA link to the MOC once per orbit.

Data streams from the NASA-provided and partner-provided spacecraft are captured by the GPM Precipitation Processing System (PPS) for processing. The PPS, developed by GSFC, is based on the existing TRMM Science Data and Information System (TSDIS), and provides for processing of science data into application and outreach products. These products include surface rain rates, calibrated brightness temperatures, and rain images.

The GVS, by comparing ground-based precipitation measurements with those obtained from space, will enable improvements in the algorithms used to process future precipitation data products.

\subsection{Core Observatory}

The Core Observatory, managed and developed by GSFC, will provide the platform for the instrument payload, including accommodating the thermal, mechanical, electrical, and data requirements of the instruments. Figure 2 shows the current layout of the spacecraft. Communication with the instruments is provided over a MLL-STD-1553 data bus. The spacecraft will be maintained in a nadir-pointing configuration, with infrequent yaw maneuvers to adjust for beta angle changes. Instrument operations require calibration maneuvers roughly once a month that involve leaving the nadir-pointing mode during orbit eclipse periods for a few orbits. Observatory power, sized for a load of 1900 Watts, is

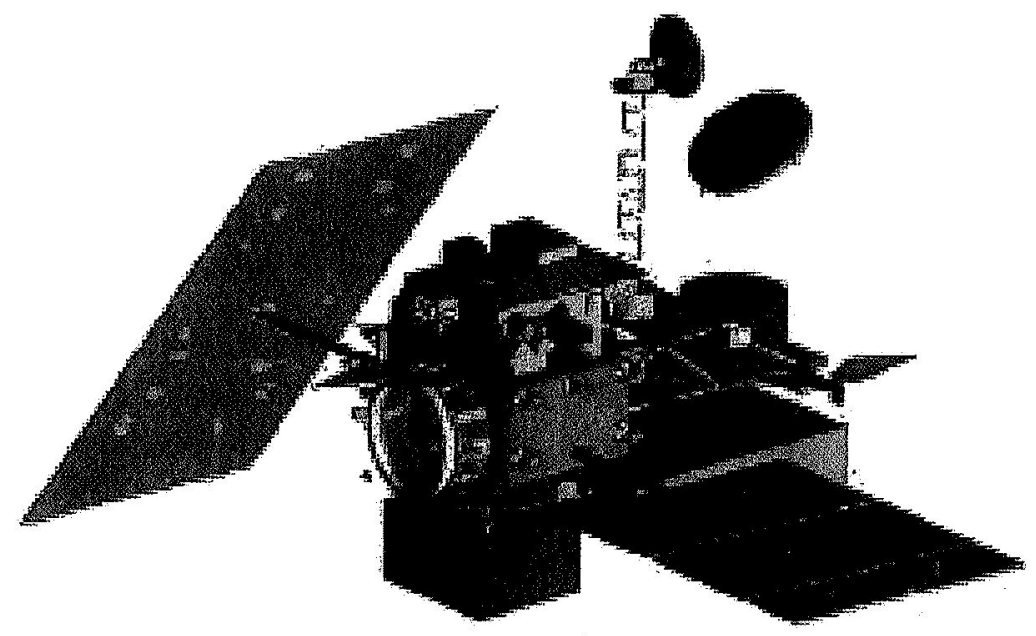

Figure 2. GPM Core Observatory Layout

provided by dual single-axis-articulated solar arrays, a Lithium-Ion Battery, and a direct energy transfer power system. The cant of the solar arrays ensures a minimum power production given any beta angle. Communication is provided via a steerable high gain antenna and two hemi-spherical omni antennas. GPS receivers on-board provide accurate orbit and time information to support the instrument geolocation requirements. A hydrazine propulsion system provides for orbit maintenance to meet the tight orbit control requirements of the DPR.

\subsection{GPM Microwave Imager (GMI)}

The GMI [3], a conically scanned microwave radiometer, is under development by Ball Aerospace, under contract to GSFC. See Figure 3. It has a $1.2 \mathrm{~m}$ aperture, and provides 13 channels over the range $10.65 \mathrm{GHz}$ to $188.31 \mathrm{GHz}$, to measure precipitation, including light rain and snow. The scanning mechanism rotates at $32 \mathrm{rpm}$, providing cross track scanning of the earth over a $1700 \mathrm{~km}$ swath with instantaneous fields-of-view (IFOVs) for the various channels ranging from $32 \mathrm{~km}$ for the $10.65 \mathrm{GHz}$ channel, down to $3.6 \mathrm{~km}$ for the $188 \mathrm{GHz}$ channel. GMI instrument parameters are listed in Table 1. 


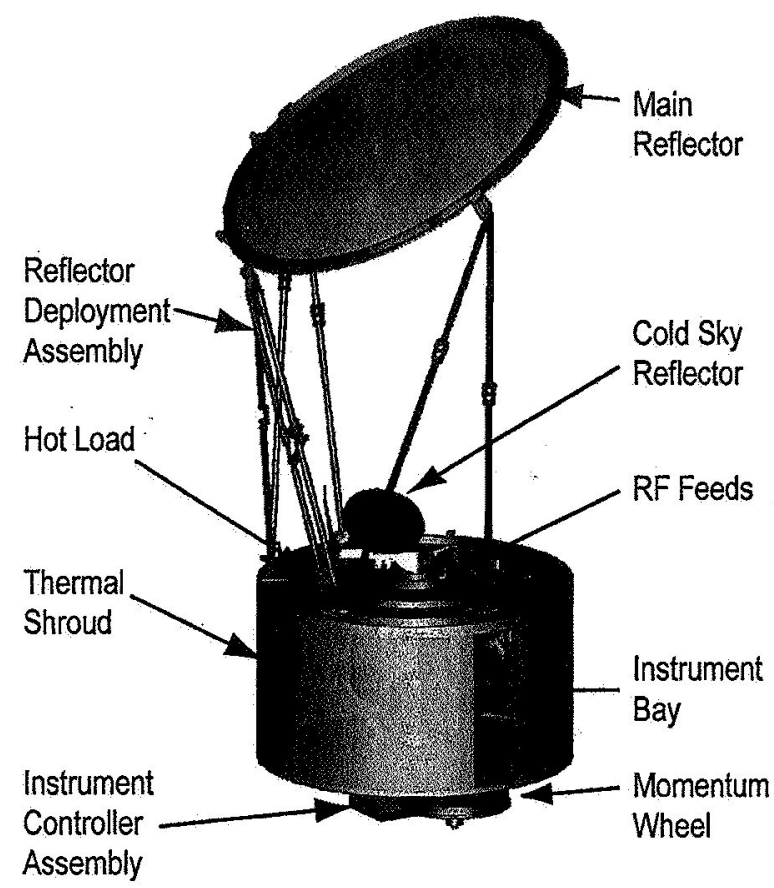

Figure 3. GPM Microwave Imager (GMI)

Table 1. GPM Instrument Parameters

\begin{tabular}{|l|c|c|c|}
\hline & DPR/KuPR & DPR/KaPR & GMI \\
\hline Type & $\begin{array}{c}148 \text { Element Active } \\
\text { Phased Array }\end{array}$ & $\begin{array}{c}148 \text { Element Active } \\
\text { Phased Array }\end{array}$ & $\begin{array}{c}\text { Passive Microwave } \\
\text { Radiometer }\end{array}$ \\
\hline Frequency & $13.6 \mathrm{GHz}$ & $35.5 \mathrm{GHz}$ & $\begin{array}{c}10.65 \text { to } 183.3 \mathrm{GHz}(13 \\
\text { channels })\end{array}$ \\
\hline Horiz Resolution & $5 \mathrm{~km}$ & $5 \mathrm{~km}$ & approx. 32 to $4.4 \mathrm{~km}$ \\
\hline Mass & $450 \mathrm{~kg}$ & $330 \mathrm{~kg}$ & $125 \mathrm{~kg}$ \\
\hline Volume & $2.4 \mathrm{mx} 2.4 \mathrm{mx0.6 \textrm {m }}$ & $1.44 \mathrm{mx} 1.07 \mathrm{mx} 0.7 \mathrm{~m}$ & $1.22 \mathrm{~m}$ Antenna dia. \\
\hline Power & $384 \mathrm{~W}$ & $326 \mathrm{~W}$ & $155 \mathrm{~W}$ \\
\hline Data Rate & $108.5 \mathrm{kbps}$ & $81.5 \mathrm{kbps}$ & $35 \mathrm{kbps}$ \\
\hline
\end{tabular}

\subsection{Dual-frequency Precipitation Radar}

JAXA and the National Institute of Information and Communications Technology (NICT) in Japan are developing the Dual-frequency Precipitation Radar (DPR) [4]. Based on the TRMM Precipitation Radar, the dual radars, one operating at $\mathrm{Ku}$-band at $13.6 \mathrm{GHz}$, and the other operating at $\mathrm{Ka}$-band at $35.5 \mathrm{GHz}$, are designed to provide three-dimensional profiles of precipitation with greater sensitivity (down to $1 \mathrm{~dB}$ ). They will also aid in the calibration of the passive microwave radiometers that make up the rest of the GPM constellation of satellites. A key driver on the design of the Core Observatory is the requirement to keep the footprints of the two radars co-aligned so that they view the same column of atmosphere. By a technique that compares the signals returned from both radars, Drop Size Distribution (DSD) of precipitation in the overlapped beams can be deduced, a key scientific objective of the mission. The dual 
radars are quite large with the KaPR $1.4 \mathrm{~m} \times 1.1 \mathrm{~m} \times 0.7 \mathrm{~m}$, and the KuPR nearly twice the size (see Figure 4). DPR instrument parameters are listed in Table 1.

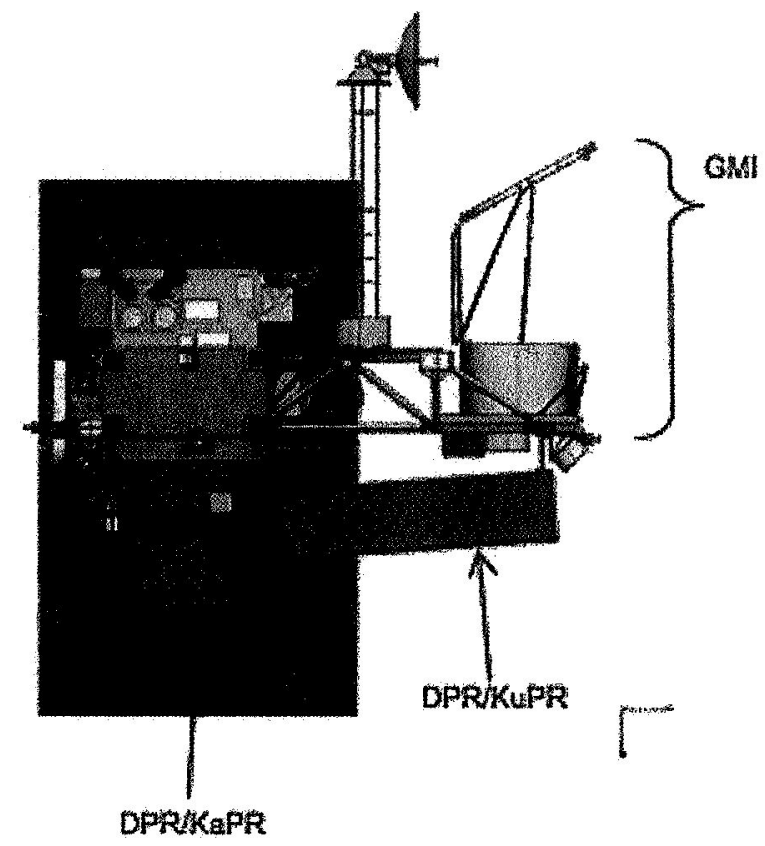

Figure 4. GPM Core Observatory showing Instrument Locations

\section{Core Spacecraft Challenges}

For the GPM mission some of the unique systems engineering challenges include orbital debris mitigation approach, spacecraft charging approach, and geolocation of instrument data. The following sections discuss the current status of the above items, all of which are the subject of ongoing study.

\subsection{Orbital Debris Mitigation}

NASA requirements for limiting orbital debris dictate that spacecraft developers show by analysis that most of the spacecraft burns up when it is predicted to re-enter the Earth's atmosphere. If considerable debris is estimated to reenter, then an option for a controlled re-entry is available. Controlled re-reentry puts many new requirements on spacecraft design, analysis, and flight operations. For this reason, GPM wanted to study an uncontrolled re-entry, where a small, but allowable quantity of material would survive the re-entry. A study was undertaken to determine which parts of the observatory would be likely to survive, and then see what could be done to modify these items. Typically, high strength metals like titanium and stainless steel are shown to largely survive the trip through the atmosphere. This is particularly true if other materials that must burn away before the atmosphere can begin ablating the surviving metal part surround the part. For GPM, a strategy was selected to first examine the design and remove these survivable materials where possible. Several areas of study were undertaken to look at increasing the likelihood that the design would "demise" before reaching the ground at the end of its mission life.

Flexures. One design area studied was the use of alternative materials in place of titanium flexures to support the large radars. Titanium has the beneficial properties of being high strength as well as thermally isolating. Analyses were performed to examine changing this interface material to aluminum, and the results of this study showed that strengthwise, the design with aluminum met the requirements of the launch loads. In the case of thermal isolation, more detailed 
analysis was performed to show that limited temperature excursions were expected, and that the design was also acceptable from a thermal point of view. The parts were redesigned with aluminum and baselined for use on the mission.

Reaction Wheels. Reaction wheels typically contain a dense, and sometimes, ablation-resistant material as the rim loading material of the wheel to maximize momentum capability while minimizing size and weight. A development effort underway at NASA GSFC has qualified a "demisable" wheel by essentially replacing the material of the wheel with aluminum, a highly demisable material. This wheel has completed environmental qualification testing, and a life test is scheduled to be underway by the end of 2006. Based on this testing, the wheels are considered a strong candidate for selection as the GPM reaction wheel. In addition, the spacecraft is laid out with the reaction wheels on the exterior of the spacecraft to allow for immediate heating from the atmosphere during reentry.

Propulsion Tank. Typical propellant tanks are constructed of titanium with an internal diaphragm to achieve propellant expulsion. For the size of tank in consideration for GPM, our initial orbital demise study indicated the tank would survive the re-entry. The study also showed that a composite overwrap pressure vessel (COPV) tank with a thin aluminum liner would demise completely. However, there are some difficulties in using an aluminum COPV tank for GPM. Typically, a space qualified COPV tank is used as a pressurant tank to hold inert gases such as nitrogen and helium. In order to be used as a liquid propellant tank, some type of propellant management device (PMD) is required to preferentially drain the propellant in zero gravity. Unfortunately, surface tension properties of untreated aluminum are quite poor compare to those of titanium and most PMD designs to date are made of titanium. Studies have shown that the aluminum surface can be treated to significantly improve its surface tension properties. Currently NASA GSFC is conducting internal research to develop a treatment process to improve aluminum's surface tension properties. This process will be the key to qualifying an aluminum COPV propellant tank for the GPM program.

An orbital debris budget has been established wherein parts are allocated "debris area" out of a total requirement. Results of on-going analyses are tracked against the existing budget, and margin exists to accommodate design changes that require non-demiseable materials. Should a development fail to achieve positive results, sufficient margin exists to fly conventional technology as a replacement.

\subsection{Spacecraft Charging}

The high inclination of GPM's orbit also poses challenges in mitigating the effects of spacecraft charging and discharging. Even though the nominal altitude is $400 \mathrm{~km}$, the $65 \mathrm{deg}$. inclination exposes the observatory to energetic electrons in the auroral regions. These auroral electrons can potentially charge the spacecraft to a few kilo-Volts and can lead to arc discharges that can disrupt spacecraft operation. Ideally, to prevent surface charging the external spacecraft surface is made at least partially conductive with a surface resistivity of less than $10^{9} \mathrm{ohms} / \mathrm{sq}$. The conductive surfaces are also tied to the common spacecraft ground to prevent differential charge buildup. However, many external surface elements of the GPM observatory are dielectric materials with higher surface resistivity than the recommended guideline. Applying a conductive coating is an option for some dielectric elements such as solar array cover glasses and MLI thermal blankets. However, for other dielectric elements such as radiator surface coatings to achieve particular thermal properties, a conductive surface coating may not be an option as it may interfere with the performance of the thermal radiator. In this case, alternative surface coatings may have to be considered.

To address the issue of charging mitigation, GPM has taken steps to make maximum the use of conductive surfaces whenever possible. This includes MLI thermal blankets, which will have a conductive outer surface for charge dissipation. In addition, all conductive elements will be tied to the common ground to minimize differential charging. For those surfaces that cannot be made conductive, GPM will address each element on a case-by-case basis aided by the results of spacecraft charging simulation software such as the NASCAP $2 \mathrm{~K}[5]$ program. NASACAP $2 \mathrm{~K}$ has a capability to simulate plasma interaction around the GEO, LEO, and polar plasma environments with realistic 3D spacecraft models. The simulation results will be used to assess charging potentials for each dielectric elements. If the results show voltages near the breakdown level for that material, it may be necessary to use differential materials of higher conductivity in order to minimize the chance of arc discharge. 


\subsection{Pointing and Alignment}

The GPM science requirements form the basis for the GPM pointing and alignment requirements. These science requirements combined with instrument characteristics drive spacecraft component selection and establish pointing allocations. The science requirements define instrument pointing requirements such as co-alignment of the KuPR and KaPR radars to facilitate drop size distribution measurements, geolocation knowledge to adequately locate the measurements, and pointing accuracy to enable a sufficient measurement height due to instrument timing issues. Pointing error is allocated to each of the subsystems and drives spacecraft component selection such as the use of star trackers for adequate pointing knowledge, GPS for ephemeris accuracy, and material selection to limit thermal excursions. As an example of this allocation process, the error budget for DPR geolocation will be shown next.

The overall geolocation requirement for $1 / 2$ pixel, translates to $2.5 \mathrm{~km}$ on the ground. For reference, this is equivalent to a 21 arc minute pointing error at an orbital height of $400 \mathrm{~km}$. The error budget, shown in Figure 2, is broken down into random and bias errors. Within the grouping of random errors, there are individual spacecraft and instrument allocations to environmental factors such as thermal distortion and launch shift, as well as pointing performance items such as attitude control system knowledge and instrument phase errors. These random errors have traditionally been root-sum-squared (RSS'd), and that is how they are handled in the GPM geolocation error budget. The total allocation for spacecraft and instrument random errors is seen to be 10.4 arc minutes.

Next, the bias errors are similarly broken down into spacecraft and instrument contributions. Bias errors are seen as onetime effects that could be removed with an on-orbit calibration program. For the geolocation error budget, gravity release, moisture desorption from composite materials, and ground alignment errors are considered bias errors. Through ground alignment activity, these types of errors are effectively removed from the overall error. Residual errors from alignment and analytical uncertainties are included as random errors, while the bias errors are listed as zero.

The random and bias errors can be summed to give an overall allocation to geolocation accuracy that is well within the requirements.

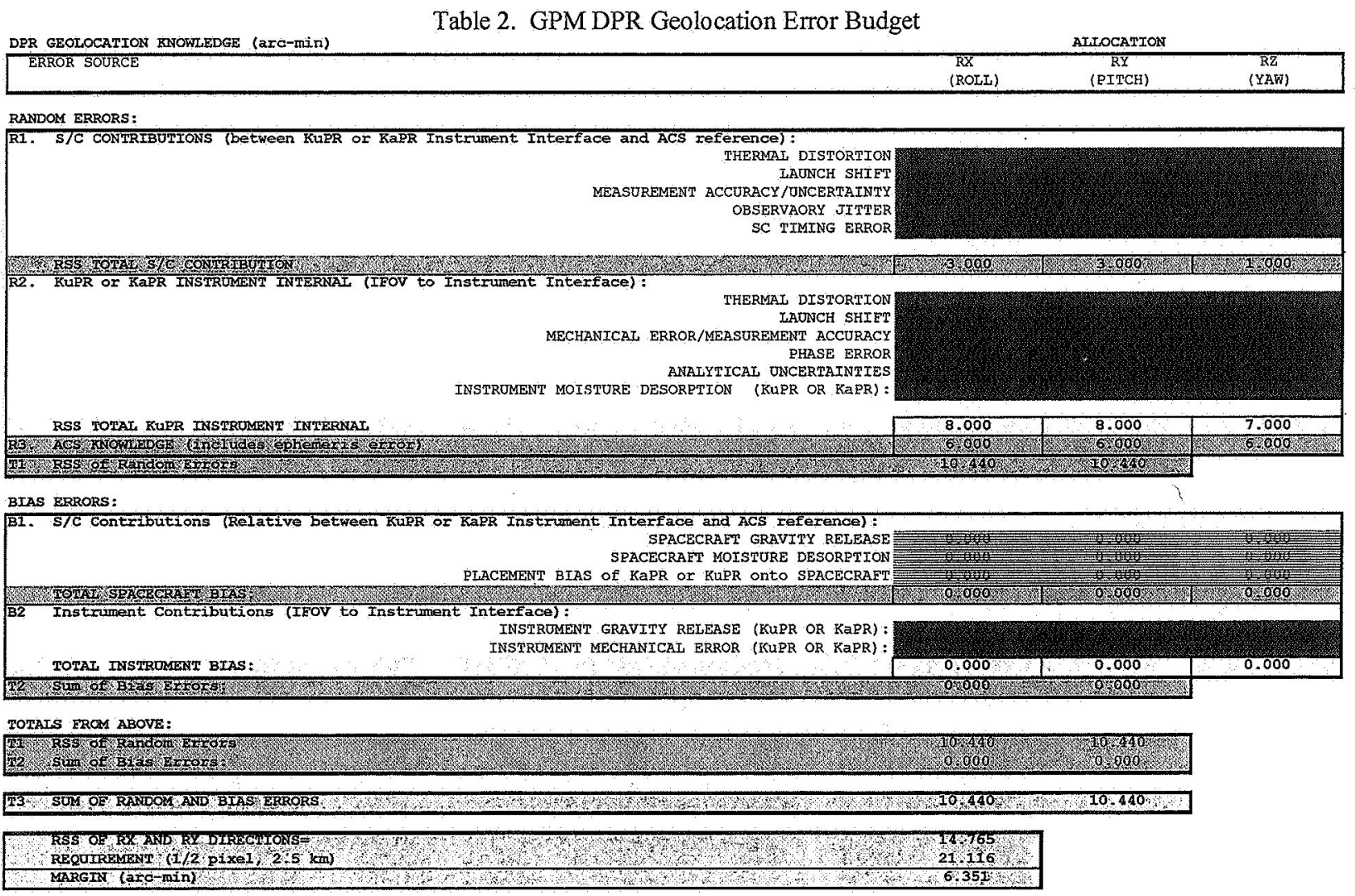




\section{Summary}

The GPM mission architecture and operations concept have been reviewed, and some of the ongoing system trade studies have been described. The in-house development team at GSFC has been assembled to meet the requirements of the Core Observatory development. Planning is underway for the GPM Mission Preliminary Design Review in 2007.

\section{REFERENCES}

1. S.P. Neeck, R.K. Kakar, SPIE Europe Remote Sensing Conference Proceedings, "Global Precipitation Measurement Development," Stockholm, Sweden, Sept. 11-14, 2006.

2. C. Kummerow, W. Barnes, T. Kozu, J. Shiue, and J. Simpson, "The Tropical Rainfall Measuring Mission (TRMM) Sensor Package" J. Atm. Ocean. Tech., Vol. 15 (3), 1998, pp. 809-817.

3. S. W. Bidwell, SPIE Europe Remote Sensing Conference Proceedings, "Global Precipitation Measurement (GPM) Microwave Imager (GMI) Instrument," Stockholm, Sweden, Sept. 11-14, 2006.

4. S. Shimizu, R. Oki, M. Kachi, M. Kojima, T. Iguchi, and K. Nakamura, "Development and Validation of Spaceborne Dual-frequency Precipitation Radar for GPM," IGARSS 2006 Conference Proceedings, Denver, CO, July 31 - Aug. 4, 2006.

5. Davis, V. A., et al., Spacecraft Charging Calculations: NASACAP-2K and SEE Spacecraft Charging Handbook, AIAA 2002-0626, 40th AIAA Aerospace Sciences Meeting \& Exhibit, January 2002 